\title{
A novel acoustic emission detection module for leakage recognition in a gas
}

\section{pipeline valve}

\author{
Zhenlin $\mathrm{Li}^{1}$, Haifeng Zhang ${ }^{2 *}$, Dongjie Tan ${ }^{2}$, Xin Chen ${ }^{1}$, Hongxiang Lei ${ }^{1}$ \\ (1,College of Mechanical and Transportation Engineering, China University of Petroleum-Beijing, Changping, Beijing, \\ 102249, China \\ 2, PetroChina Pipeline R\&D Center, Langfang, Hebei 065000, China)
}

\begin{abstract}
Internal valve leakage in a natural gas pipeline seriously impairs the safe operation on pipelines, and the recognition of leakages has therefore been a major concern of the industry. In this study, a novel leakage detection scheme based on kernel principal component analysis (kernel PCA) and the support vector machine (SVM) classifier for the recognition of the leakage level is constructed. Using this approach, the acoustic signal of the leakage is obtained as the feature source using an acoustic emission (AE) sensor. The kernel PCA is used to reduce the dimensionality of the features and extract the optimal features for the classification process, and the SVM is applied to perform the recognition of the leakage levels. The performance of the classification process based on kernel PCA and the classifier are evaluated in terms of the accuracy, Cohen's kappa number and training time. The experimental results demonstrate that the intelligent recognition model based on kernel PCA and SVM classifier is very effective for recognizing the leakage level of a valve in a natural gas pipeline.
\end{abstract}

Keywords: Valve leakage recognition; Acoustic emission; Kernel principal component analysis; Support vector machine.

\section{Introduction}

The inevitable events of gas pipeline valve leakage during the gas transportation process pose serious problems to the availability, reliability, and economy of the pipeline. Thus, the possibly used safety measures focused on early detection of both small and large leakages is not only a guarantee for safer operation but also a help for reducing the costs of the industry. To monitor the valve yield condition for substantial cost savings and safer working conditions, a great number of methods have been developed. However, the currently available leakage detection methods provide little capability for the quantitative recognition of leakage levels. 
One common measurement technique for measuring the leakage of valves is acoustic emission (AE) (Sharif et al. 1998), which is a new non-destructive testing (NDT) technique with very high sensitivity that can be used to detect weak signals without hindering operations (Christian et al., 2008). A comprehensive review of the development history of acoustic emission was provided by Drouillard (1996). In recent years, researchers have studied the characteristics of AE signals in the process of valve leakage. Lee et al. (2006) provided a detailed summary of the acoustic emission signals indicating different characteristics of the check valve. Kaewwaewnoi et al. (2010) investigated the relationship between the internal fluid leakage of a valve and the acoustic emission signal, and a further study has been made by Prateepasen et al. (2011) for the development of intelligent portable noninvasive instruments. Meland et al. (2011) analyzed the frequency spectrum of internally leaking shutdown valves. All of the above studies indicated that the leakage of pipelines can be detected qualitatively using AE technology. On the other hand, it is also important to measure the leakage level quantitatively for the safer operation of the natural gas pipeline. In this study, an intelligent leakage recognition method for valve leakages in a gas pipeline using acoustic emission was developed. The process of intelligent leakage recognition includes the dimensionality reduction of the acoustic signal and the classification of the leakage. As a pattern recognition method, the support vector machine (SVM) has been extensively employed to solve classification and regression problems (Cortes and Vapnik, 1995). The SVM has been successfully used in many fields, including valve leakage (Yang et al., 2005), mechanical fault diagnosis (Oh et al., 2009), image and video processing (Ding et al., 2008), medical engineering (Gil et al., 2010), and chemical engineering (Kulkarni et al., 2005). To improve the accuracy of classification, several feature parameters were calculated in the time and frequency domains. Because large numbers of features may lead to the reduction of the dimensionality and thereby reduced performance of classification, in this study, the kernel principal component analysis (kernel PCA) based on the non-linear feature of the reduction of dimensionality was adopted to convert the existing features into a space of a smaller number of dimensions and thereby improve the classification performance.

In the present study, an intelligent model was constructed to quantitatively recognize the leakage level of valves in gas pipelines. The acoustic parameter features of different leakage levels were first obtained using an acoustic emission sensor. The kernel PCA was then employed to transform the features into a lower-dimensional space and extract the effective features. Typical kernel functions of important parameters used for indicating the performance of the kernel PCA were compared and analyzed. Then, the effective features were trained and tested using the SVM to determine the level of valve leakage. To verify the SVM models, the performance of the classifiers, including the 
accuracy, the Cohen's kappa number, and the training time, were compared to the corresponding data from the $k$-nearest neighbor classifier ( $k$-NN) (Liao et al., 2002), neural network classifier (NN) (Yu et al., 2006), naive bayes classifier (NB) (Jiang et al., 2012), and decision tree classifier (DT) (Sethi, 1997).

\section{Methods}

\subsection{Acoustic emission in valve application}

Acoustic emission is a spontaneous release of elastic energy during the deformation of a material and can be detected by an AE sensor in all directions. The main sources of acoustic emission are plastic deformation, crack growth, leakage, burning and fracture (Malhotra, 2003). Therefore, the acoustic signal induced by the internal leakage of the pipeline valve is a typical acoustic emission phenomenon. Gas leakage in the valve introduces a pressure difference in the gas space and then generates turbulence. The turbulence not only disturbs the normal flow of gas but also leads to an interaction between the frequency elastic wave (acoustic emission signal) and the valve wall. The leakage information spreads from the elastic wave to the surface of the valve, and the AE sensor can be used to pick up the acoustic emission signals. The degree of the pipeline valve leakage can then be evaluated through signal analysis and processing.

In signal analysis and processing, different parameters can be extracted from the signals. The AE signals of leakage are continuous waveforms that can be analyzed by several special techniques. In this study, the measured AE parameters selected to identify the leakage in the time and frequency domains are listed in Table 1.

Table 1 Measured AE parameters for leakage recognition

\begin{tabular}{ll}
\hline Time domain & Frequency domain \\
\hline Mean value & Root variance frequency \\
Standard deviation & Peak value \\
RMS & Frequency center \\
Energy & \\
Entropy & \\
\hline
\end{tabular}

The AE signal is very useful for studying the characteristics of valve leakage mechanism, because the measured AE parameters can be directly related to the level of leakage. Relative studies on valve frequency analysis showed that the AE signal obtained from valve leakage is usually in the frequency range of 100 300 kHz (Noipitak et al., 2011). The peak value and frequency center of the AE gradually increases with the leakage level. Consequently, the occurrence and levels of valve leakage can be evaluated by the parameters of the AE signal features in both the time and spectral domains. 


\subsection{Kernel principal component analysis (kernel PCA)}

Principal component analysis (PCA) is probably the best known of the techniques of multivariate analysis, it's central idea is to reduce the dimensionality of data set in which there are large number of variables. The internal model of PCA is linear relationship, it focuses on the two order correlation between variables, which deals with the two order correlation between variables. PCA is less effective to extract the nonlinear characteristics of the acoustic parameters of leakage in valve, therefore, Kernel PCA is proposed to extend the feature extraction method in the nonlinear case. The kernel principal component analysis is constructed from the traditional linear PCA in a high-dimensional space using a kernel function. Using the kernel PCA, the principal eigenvectors of the kernel matrix rather than those of the covariance matrix should be calculated. The reconstruction of the traditional PCA in kernel space is straightforward because of the similarity between the kernel matrix and the inner product of the data points that are used to construct the kernel function. The properties of constructing nonlinear mappings for kernel PCA can be provided by the application of PCA in the kernel space (Cho et al., 2005).

The idea of kernel PCA is to obtain linear operations by mapping the original non-linear input vectors $x_{k}$ to a high-dimensional feature space $\phi\left(x_{k}\right)$ using a kernel function. A set of centered observed values, i.e., $x_{k}(k=1 \ldots M$, and $\left.\sum_{k=1}^{M} x_{k}=0\right)$ are given for the kernel PCA model construction, and a multi-dimensional feature space is adopted. According to nonlinear mapping, namely, $\phi: R_{N} \rightarrow F$, the observed values in the current space are converted into a high-dimensional feature space $\phi\left(x_{k}\right)$. The sample covariance matrix $\tilde{C}$ in the feature space can be expressed by

$$
\tilde{C}=\frac{1}{M} \sum_{k=1}^{M} \phi\left(x_{k}\right) \phi\left(x_{k}\right)^{T}
$$

The eigenvalues and eigenvectors of the covariance matrix $\tilde{C}$ in feature space $F$ can be calculated by the PCA solver as

$$
\lambda v=\tilde{C} v=\frac{1}{M} \sum_{k=1}^{M}\left(\phi\left(x_{k} \cdot v\right)\right) \phi\left(x_{k}\right)
$$

In the above equation, $\lambda$ and $v$ are an eigenvalue and eigenvector of the covariance matrix, respectively. All of the solutions for $v$ with $\lambda \neq 0$ lie in the span of $\phi\left(x_{1}\right) \sim \phi\left(x_{\mathrm{M}}\right)$. A set of coefficients $\alpha_{i}(i=1, \ldots M)$ that meet the following conditions exist, i.e.,

$$
v=\sum_{i=1}^{M} \alpha_{i} \phi\left(x_{i}\right)
$$


Combining Eqs. (2) and Eq.(3), the following equation can be obtained:

$$
\lambda\left(\phi\left(x_{k}\right) \cdot v\right)=\phi\left(x_{k}\right) \cdot \tilde{C} v, k=1, \ldots M .
$$

By defining an m-dimensional matrix $K$ as $K\left(x_{i}, x_{j}\right)=\left(\phi\left(x_{i}\right) \cdot \phi\left(x_{j}\right)\right)$, a kernel function equal to the inner product of vectors $\phi\left(x_{i}\right)$ and $\phi\left(x_{j}\right)$, which are the corresponding values of $x_{i}$ and $x_{j}$ in the high-dimensional feature space, respectively, can be obtained. Three typical kernel functions are listed in Table 2, and the kernel function can be written in a simplified form as:

$$
M \lambda \alpha=K \alpha,
$$

where $\alpha$ denotes a column vector with its components $\alpha_{1}, \ldots \alpha_{M} . \lambda_{I} \leq \lambda_{2} \leq \ldots \leq \lambda_{M}$ are eigenvalues of $K$ with $\lambda_{p}$ the first nonzero eigenvalue (assume $\phi \neq 0$ ), and $\alpha^{l}, \alpha^{2}, \ldots, \alpha^{M}$ are the corresponding eigenvectors. $\alpha^{p} \sim \alpha^{M}$ can be normalized by requiring the corresponding vectors in the feature space $F$ to be normalized, namely,

$$
v^{k} \cdot v^{k}=1, k=p, \ldots M .
$$

The above equation can be changed into a normalization condition through a combination of Eq. (3) and Eq. (5), i.e.,

$$
1=\sum_{i, j=1}^{M} \alpha_{i}^{k} \alpha_{j}^{k}\left(\phi\left(x_{i}\right) \cdot \phi\left(x_{j}\right)\right)=\sum_{i, j=1}^{M} \alpha_{i}^{k} \alpha_{j}^{k} K\left(x_{i}, x_{j}\right)=\left(\alpha^{k} \cdot K \alpha^{k}\right)=\lambda_{k}\left(\alpha^{k} \cdot \alpha^{k}\right) .
$$

The principal components $x_{k}$ are calculated based on the estimated value of $\alpha_{i}^{k}$ according to

$$
S_{k}(i)=\left(v^{k} \cdot \phi(x)\right)=\sum_{i=1}^{M} \alpha_{i}^{k} K\left(x_{i}, x_{k}\right), i=1, \ldots M .
$$

Note that the maximum number of principal components in the kernel PCA is $M$. In summary, the necessary steps for calculating the principal components are as follows:

(1) Compute the matrix $K$.

(2) Calculate the eigenvectors and normalize matrix $K$ in feature space $F$.

(3) Compute the projection of an experimental point onto the eigenvectors.

Table 2 Formulation for kernel function

\begin{tabular}{ll}
\hline Kernel function & $K\left(x_{-i}, x_{-j}\right)$ \\
\hline Linear & $x_{-i} \cdot x_{-j}$ \\
Polynomial & $\left(x_{-i} \cdot x_{-j}+1\right)^{d}$ \\
RBF & $\exp \left(-\left\|x_{-i}-x_{-j}\right\|^{2} / 2 \sigma^{2}\right)$ \\
\hline
\end{tabular}




\subsection{Support vector machine classifier for recognition}

The support vector machine method was first proposed by Cortes and Vapnik in 1995, and the method can be classified to a new category of methods with good generalization based on the Vapnik-Chervonenkis dimension and the structure risk minimization principle. Compared to traditional statistics, the SVM shows superior performance, especially for problems with a small number of samples, nonlinearity and high dimensionality. Its main advantage lies in finding the optimal compromise between the complexity of the model and the learning ability to obtain the best generalization. The key idea of SVM is to map the original pattern space into a high-dimensional feature space through non-linear mapping functions, and in this way, the optimal separating hyper-plane can be constructed in the feature space. Consequently, a non-linear problem in low-dimensional space can be transformed into a linear problem in high-dimensional space (Cortes and Vapnik, 1995).

Binary classification is the basis of multi-classification problems and can be used as an example to further explain the principle of SVM. Assume that a training dataset is composed of an input vector $X_{i}\left(i=1, \ldots l, X_{i} \in R^{d}\right)$ of length $n$ with the class label $y_{i} \in[-1,+1]$. For the binary classification, the linear separating hyper-planes for the detachable case is shown in Fig.1.

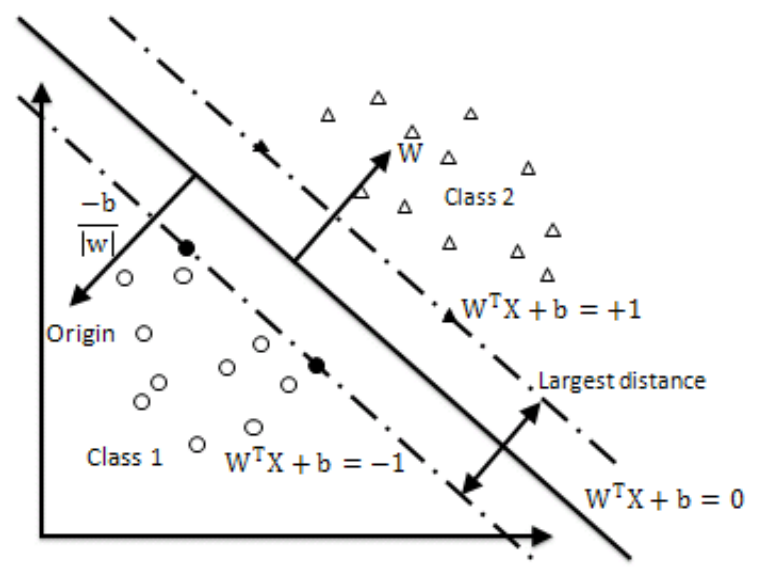

Fig.1 Linear separating hyper-planes for the separable case

The points $X$ at the boundary of the hyper-plane satisfy the condition $W^{T} X+b=0$, where $W$ is a vector normal to the hyper-plane. $|b| /\|W\|$ is the perpendicular distance from the hyper-plane to the origin, with $\|W\|$ the Euclidean norm of $W$. Because the goal of the SVM is to find the largest possible distance between the hyper-plane boundary $\left(W^{T} X+b=0\right)$ and the training data, $W$ and $b$ must be set to satisfy the conditions that the distance between the two marginal lines be the maximum, namely, $W^{T} X+b=-1$ and $W^{T} X+b=1$. For the linearly detachable case, the SVM algorithm simply searches for the separating hyper-plane with the largest distance. Constructing the optimal 
separating hyper-plane with the largest distance can therefore be generalized to the problem (Burges, 1998)

$$
\underset{W, b}{\arg \min }\left\{\frac{1}{2} W^{T} W\right\}
$$

which is subject to the following equation condition:

$$
\begin{array}{ll}
W^{T} X+b \geq+1 \quad \text { for } y_{i}=+1 \\
W^{T} X+b \leq-1 \quad \text { for } y_{i}=-1
\end{array}, i=1, \ldots l .
$$

The above equation can be rewritten in the following form:

$$
y_{i}\left(W^{T} X+b\right) \geq 1, i=1, \ldots l .
$$

However, because there are no linearly separable structures for most of the distributions, the above equation is unsolvable. To overcome this issue, Cortes and Vapnick introduced the slack variable $\xi_{i}\left(\xi_{i} \geq 0, i=1, \ldots l\right)$ to modify Eq.(9) to be (Choi, 2003)

$$
\underset{W, b, \xi}{\arg \min }\left\{\frac{1}{2} W^{T} W+C \sum_{i=1}^{l} \xi_{i}\right\}
$$

The above equation can also be expressed in another form, i.e.,

$$
\begin{array}{ll}
W^{T} X+b \geq+1-\xi_{i} & \text { for } y_{i}=+1 \\
W^{T} X+b \leq-1+\xi_{i} & \text { for } y_{i}=-1
\end{array}, i=1, \ldots l,
$$

or

$$
y_{i}\left(W^{T} X_{i}+b\right) \geq 1-\xi_{i}, \xi_{i} \geq 0, i=1, \ldots l .
$$

In Eq. (12), $\xi_{i}$ is the slack variable and $C$ is a penalty factor. The target function can be transformed into a dual problem using the convex quadratic programing method according to optimization theory. Using the Lagrange function to remove $W$ and $b$, the dual problem can be reduced to

$$
\min \left\{\frac{1}{2} \sum_{i=1}^{l} \sum_{j=1}^{l} \alpha_{i} \alpha_{j} y_{i} y_{j} \phi\left(x_{i}\right)^{T} \phi\left(x_{j}\right)-\sum_{i=1}^{l} \alpha_{i}\right\}
$$

where

$$
0 \leq \alpha_{i} \leq C, i=1, \ldots l, \sum_{i=1}^{l} y_{i} \alpha_{i}=0
$$

Eqs. (15) and (16) describe the quadratic programing problems with inequality constraints and have unique solutions. According to the functional theory, there is an inner product function satisfying the Mercer conditions, i.e., $\phi\left(x_{i}\right)^{\mathrm{T}} \phi\left(x_{j}\right)=K\left(x_{i}, x_{j}\right)$, where $K\left(x_{i}, x_{j}\right)$ is called the "kernel function" (with the same structure as the kernel PCA 
kernel function). In this study, the kernel functions were chosen empirically, and the optimal results were achieved using the RBF kernel function. Vectors for which $\alpha_{i}>0$ are called support vectors after solving the dual problem. The classification decision-making function can be written as a sign function, namely,

$$
f(x)=\operatorname{sign}\left[\sum_{i=1}^{l} y_{i} \alpha_{i} K\left(x_{i}, x_{j}\right)+b^{*}\right]
$$

where $l$ is the number of support vectors and $b^{*}=y_{i}-\sum_{i=1}^{l} y_{i} \alpha_{i} K\left(x_{i}, x_{j}\right)$ is the classification threshold. Subsequently, a classification problem is transformed into a quadratic programming problem by the SVM.

The above discussion addresses the binary classification where the labels have only two values, namely, 1 and -1 . However, in the present study, not only the location of a valve leakage but also the leakage level is a concern in engineering practice. Thus, the multi-class problems should further be considered. Several multi-SVM methods have been used for multi-classification, such as the one-against-all, one-against-one, hierarchical, and directed acyclic graph SVMs (Tsujinishi et al., 2003; Tohmé et al., 2011). The computational time of the one-against-one method is much shorter than that of other methods, making it more suitable for practical applications (Hsu et al., 2006). In this study, the one-against-one method, which constructs $k(k-1) / 2$ SVM models, is employed to recognize the leakage of gas pipeline valves. Each of the models is trained by data from two classes, and $k(k-1) / 2$ is the number of classes. Using the training data of the $m_{t h}$ and $n_{t h}$ classes, Eqs. 12 14 can be, respectively, modified to

$$
\begin{aligned}
& \underset{W^{m n}, b^{m n}, \xi^{m n}}{\arg \min }\left\{\frac{1}{2}\left(W^{m n}\right)^{T} W^{m n}+C \sum_{i=1}^{l} \xi_{i}^{m n}\right\}, \\
& \left(W^{m n}\right)^{T} X+b^{m n} \geq+1-\xi_{i}^{m n} \text { for } y_{i}=m \\
& \left(W^{m n}\right)^{T} X+b^{m n} \leq-1+\xi_{i}^{m n} \text { for } y_{i}=n
\end{aligned}
$$

and

$$
\xi_{i}^{m n} \geq 0, i=1, \ldots l .
$$

After all $k(k-1) / 2$ classifiers have been constructed, methods are available for the further testing. The voting method, which is also known as the max-win strategy, is the foremost one. Using the voting method, if the testing vector $X_{i}$ is in the $m_{t h}$ class, the vote for the $m_{t h}$ class is incremented by one. Otherwise, the $n_{t h}$ class is incremented by one. At the end of voting, the class with the largest number of votes is chosen as the most suitable class.

\subsection{Performance evaluation}

To evaluate the classification performance of different structures including characteristic parameters and kernel 
functions, confusion matrix measures containing the overall accuracy and the Cohen's kappa number were employed. The overall accuracy is the classification correct ratio of the whole set of data, which is equal to the number of correct classifications divided by the total classification number. Cohen's kappa is another statistical measurement used to evaluate the classification accuracy and can be expressed by

$$
\text { Cohen's kappa }=\frac{\operatorname{Pr}(a)-\operatorname{Pr}(e)}{1-\operatorname{Pr}(e)},
$$

where $\operatorname{Pr}(a)$ is the relative observed agreement among raters and $\operatorname{Pr}(e)$ is the forecast probability of chance agreement. Note that the forecast and actual results are in complete agreement if Cohen's kappa $=1$. On the contrary, if there is no agreement (beyond chance) between the results, the value is zero. The detailed calculation process can be found in Cohen (1960).

\section{Intelligent valve leakage recognition experiment}

\subsection{Leakage recognition methodology}

In this study, the acoustic emission signal of the valve leakage is acquired for valve leakage recognition. The leakage recognition methodology consists of data acquisition, signal processing, feature computing, feature extraction, classification processing, and solution output. The novel valve leakage recognition methodology, which is based on acoustic emission technology, the kernel PCA, and SVM multi-class classification, is depicted in Fig.2. The procedure of the methodology can be summarized in the following steps:

Step 1: Acoustic emission sensors are installed on the upstream and downstream pipes near the ball valve to obtain the acoustical signal.

Step 2: The FFT/IFFT is used to eliminate the interference noise from the original AE signal to perform the signal processing.

Step 3: The feature computing is extracted using statistical feature parameters from the time domain and frequency domain.

Step 4: The kernel PCA is applied to reduce the dimensionality and obtain the optimal features in the feature extraction process.

Step 5: The classification of leakage levels is carried out by the SVM classifier.

Step 6: The result of the valve leakage recognition is obtained. 


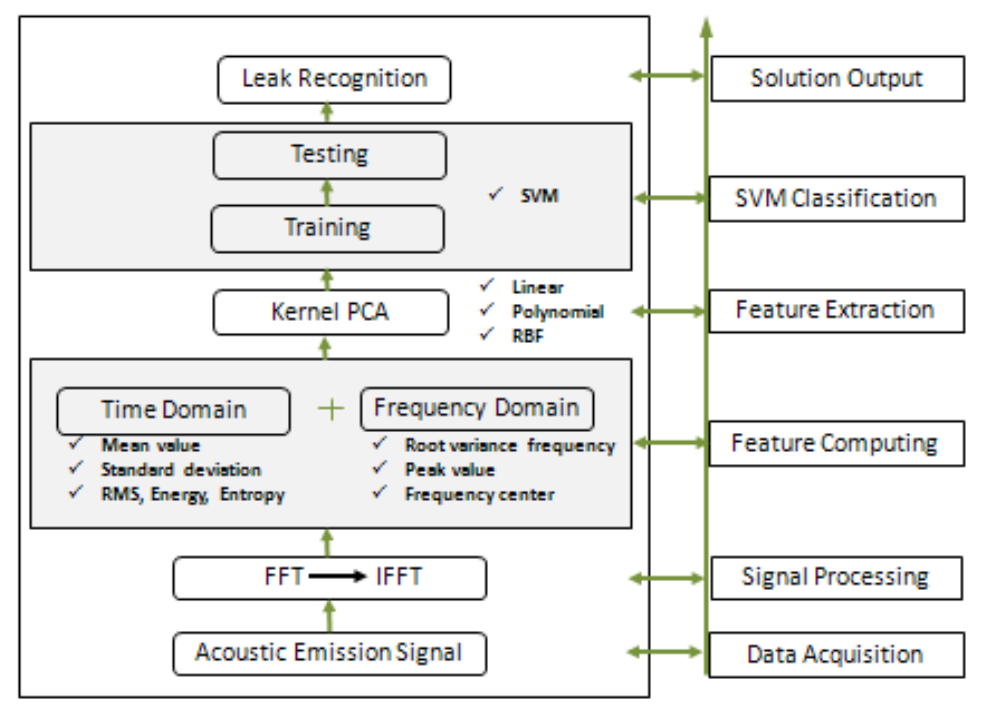

Fig.2 Flowchart of valve leakage recognition methodology

\subsection{Setup and process of the experimental system}

The experimental schematic of the acoustic emission system is shown in Fig.3. A gas cylinder with a volume of $40 \mathrm{~L}$ was used to provide the leakage air, and a DN50 ball valve was the experimental object, with a bearing capacity of 1.5 MPa. A flowmeter was used to measure the gas leakage rate through the valve. In the acoustic emission system, a wide-band $\mathrm{AE}$ sensor (SR150N) that covers the frequency range from 20 to $220 \mathrm{kHz}$ was used to detect the AE signals of the gas pipeline valve leakage. A $40 \mathrm{~dB}$ pre-amplification was employed to amplify the AE signal. A data acquisition unit with a $1 \mathrm{MHz}$ sampling rate and 24 bit $\mathrm{A} / \mathrm{D}$ conversion was adopted in the experiment. During the process of the experiment, the AE signal was processed by high-pass filtering with a cut-off frequency of $20 \mathrm{kHz}$ of to reduce the impact of the environmental noise during the detection.

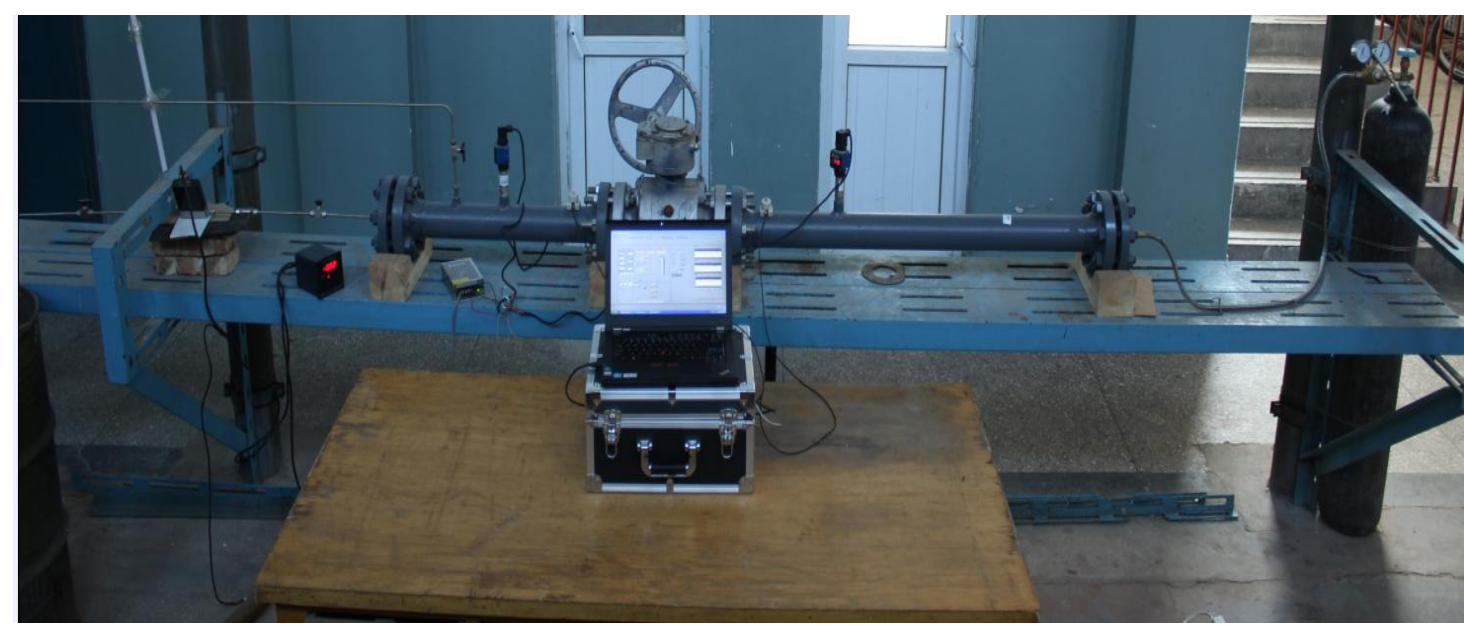

Fig.3 Experimental platform for the detection of valve leakage 
The gas cylinder was a source of compressed gas that was regulated through the regulating valve and flowed through the upstream piping, valve, and downstream piping before finally being discharged into the atmosphere through the flowmeter. The sensor was fixed on the center of the outer wall of the valve, and the DN50 ball valve was a damaged valve with internal leakage. In this paper, the SVM algorithm was applied to identify the levels of different internal leakage flow, so the labels of the internal leakage flow levels of the valve should be set. Due to the no relevant quantitative criteria used to define these levels of natural gas pipeline valve leaks ( e.g. accidental or major leaks) in a long running process at present, eight levels (level 1 to level 8) of internal leakage flow are self-defined in this experiment, level1:(0-1)SLM (standard liters per minute), level2: (1-2)SLM, level8:(7-8)SLM respectively, and the eight levels of leakage for recognition were produced by adjusting the pressure-regulating valve. The valve leakage rate and $\mathrm{AE}$ signal were recorded every second at each leakage level with the sampling rate of the $\mathrm{AE}$ signal waveform equal to $1 \mathrm{MHz}$. Experimental parameters were recorded 100 times per level, and the total number of records is 800 . The collected data were analyzed using the intelligent leakage recognition system. Each algorithm was simulated at the same time using the PRTools Matlab toolbox for the reduction of dimensionality.

\subsection{Results and discussion}

\subsubsection{Feature dimension reduction}

A total number of 6400 features (i.e., 8 parameters, 100 signals, and 8 levels) for the intelligent recognition of leakage are considered. Among the eight feature parameters, five are in the time domain and the rest are in the frequency domain. The feature parameters are shown in Table 1.

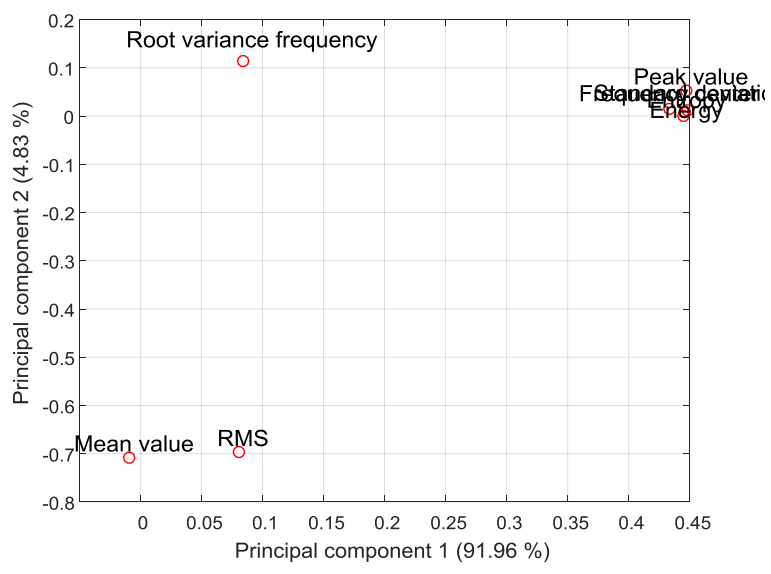

Fig.4 Circle of correlations for measured AE parameters 
The original features are disordered and redundant and therefore cannot be used to distinguish the different levels of leakage. The kernel PCA is applied to analyze the correlation of the components, so as to achieve the purpose of reducing the amount of data storage and eliminating the useless data. The circle of correlations for measured AE parameters is presented in Fig.4, and the results show that these variables (Standard deviation, Energy, Entropy, Peak value and Frequency center) have a great relationship with each other. Therefore, using the kernel PCA function, the characteristics with high dimension can be mapped in to a low dimension as a new feature to realize feature extraction, and a better model is obtained if there are kept only the features correlated with the two components.

To avoid degrading the performance of the classifier, the kernel PCA was adopted to reduce the dimensionality and thereby extract the effective features. The first two principal components are plotted in Fig.5, showing the results using the linear kernel function (Fig.5 (a)) and the polynomial kernel function (Fig.5 (b)), in which the kernel parameters $d$ and $R$ are equal to 1 and 3, respectively. In Fig.5 (c), the RBF kernel function with the parameter $\sigma$ equal to 10 was employed in the kernel PCA. It can be seen that the clusters of the eight levels of leakage are well separated from each other. However, the performance of the kernel PCA with the polynomial kernel function is not superior to those of the linear and RBF kernel functions, as was expected. As shown in Fig.5 (b), there are some overlaps, especially between the leakage levels lower than 6. However, using the other two functions, all eight levels of leakage are well separated from each other.

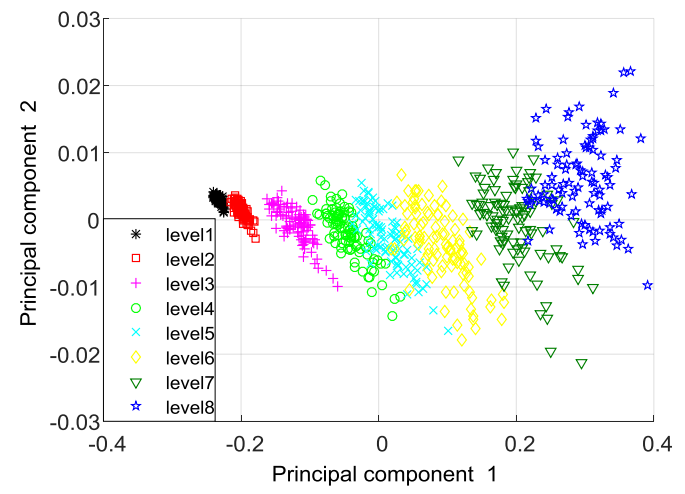

(a) Linear kernel function 


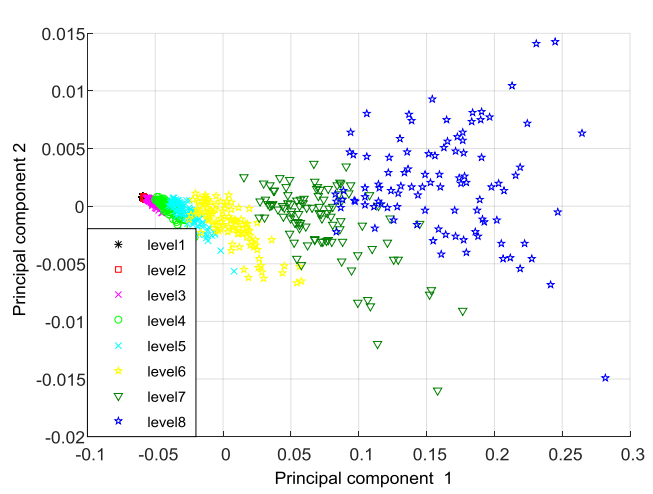

(b) Polynomial kernel function

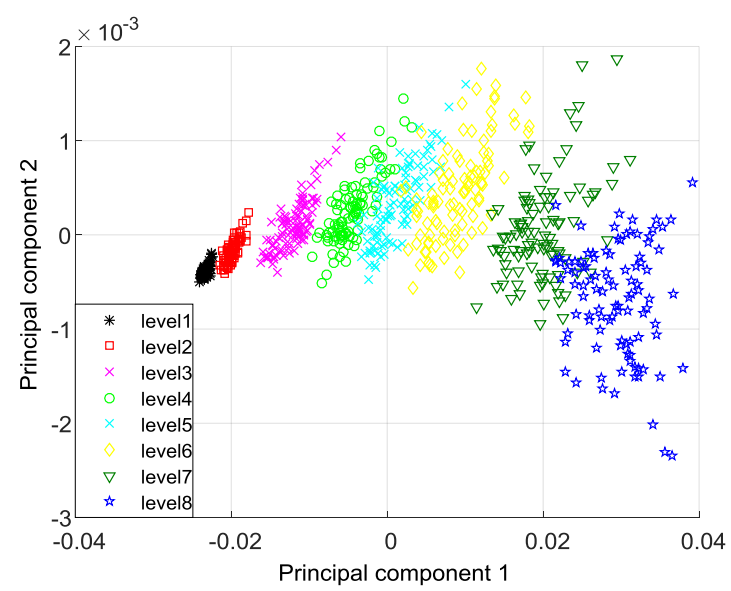

(c) RBF kernel function

Fig.5 Feature dimension reduction using kernel PCA: (a) kernel PCA with linear kernel function, (b) kernel PCA with polynomial kernel function, (c) kernel PCA with RBF kernel function

The correlation coefficients among variables and principal components are shown in Table 3, as it can be seen that the correlations of the variables (expect the mean value and frequency center ) with the first component scores, with component accounts for over $90 \%$ of the data's variance. The kernel PCA could be effectively used for the reduction of the feature dimensionality.

Table 3 Correlation coefficients among variables and principal components

\begin{tabular}{|l|r|r|r|r|r|r|}
\hline & \multicolumn{7}{|c|}{ principal components } \\
\hline Variables & Prin1(linear) & Prin2(linear) & Prin1(poly) & Prin2(poly) & Prin1(RBF) & Prin2(RBF) \\
\hline Mean value & -0.141 & 0.503 & 0.198 & 0.309 & -0.141 & -0.592 \\
\hline Standard deviation & 0.988 & 0.150 & 0.940 & 0.008 & 0.988 & -0.149 \\
\hline RMS & 0.991 & 0.128 & 0.928 & -0.005 & 0.991 & -0.123 \\
\hline
\end{tabular}




\begin{tabular}{|l|r|r|r|r|r|r|}
\hline Energy & 0.935 & 0.300 & 0.980 & 0.138 & 0.935 & -0.320 \\
\hline Entropy & 0.983 & 0.015 & 0.959 & -0.098 & 0.983 & 0.004 \\
\hline $\begin{array}{l}\text { Root variance } \\
\text { frequency }\end{array}$ & 1.000 & -0.002 & 0.921 & -0.128 & 1.000 & 0.001 \\
\hline Peak value & 0.999 & -0.019 & 0.909 & -0.135 & 0.999 & 0.022 \\
\hline Frequency center & 0.121 & -0.062 & 0.079 & -0.037 & 0.121 & 0.072 \\
\hline
\end{tabular}

\subsubsection{Training and classification}

The feature dimensionality reduction method using the kernel PCA is divided into two parts, i.e., training and testing. 50\% of the selected features are employed for training, and the remaining 50\% are used for evaluating the performance of the classifiers. In this study, the one-against-one SVM multi-classification was applied to perform the recognition process, and the RBF kernel was selected as the basic kernel function. There are two parameters associated with this kernel to determine the performance of the SVM, namely, $C$ and $\gamma \cdot C$ is the penalty factor, and $\gamma$ is the kernel parameter. The best group of both parameters $(C, \gamma)$ thus needs to be identified to optimize the performance of the SVM classification. The cross-validation method was adopted to calculate the optimum parameter group, and the complete results of the parameters selected for the training features that were extracted from the kernel PCA are $(C=11, \gamma=4)$ for the linear kernel function, $(C=16, \gamma=16)$ for the polynomial kernel function, and $(C=65, \gamma=11)$ for the RBF kernel function. After the optimal parameter groups are determined, the whole training feature dimensionality reduced by the kernel PCA is trained again to establish the final classifier using the SVM. In this paper, the one-against-one SVM multi-classification is used to classify the 8 levels $(k=8)$ of the inner leakage, which constructs $k(k-1) / 2$ SVM classifiers, so a total of 28 SVM classifiers are required in this model and in turn corresponds to $1-2,1-3,1-8, \ldots, 2-3,2-8, \ldots, 7-8 \mathrm{SVM}$ classifier. Training through 400 groups of samples processed using the linear kernel PCA, the equation of the 1-2SVM class separation line on the internal leakage level1 and level 2 is obtained, i.e., $f(x)=\operatorname{sign}\left[\sum_{i=1}^{l} y_{i} \alpha_{i} K\left(x_{i}, x_{j}\right)+b^{*}\right]$, where $x_{i}$ (support vector)=[0.02; 0.55], [0.03; $0.52], \ldots \ldots$, its number $l=17$, the $w_{i}=y_{i} \alpha_{i}=11.31,1.89, \ldots \ldots$, and $b^{*}=0.054$. Due to the length of the article, the equations of the other 27 class separation lines are not listed here. In the end, for the training features processed with linear kernel PCA, a total of 161 support vectors of the 28 SVM classifiers are calculated to classify the 8 leakage levels, 184 support vectors are calculated for the polynomial kernel PCA, and 190 support vectors for RBF kernel PCA. The number of support vectors reflects the complexity of the SVM model, and the calculated number 
proves that there is no obvious difference between the three kernel functions in the kernel PCA for the establishment of SVM mode in the experiment. The class separation lines and of the training features using the SVM classifier are plotted in Fig.6, and the separation lines explain that the eight levels of leakage are segmented clearly by the SVM classifier. Especially for the training features with polynomial kernel PCA in Fig.6(b), SVM also has a very good classification effect on the indistinguishable internal leakage levels of level 1 to level 4.

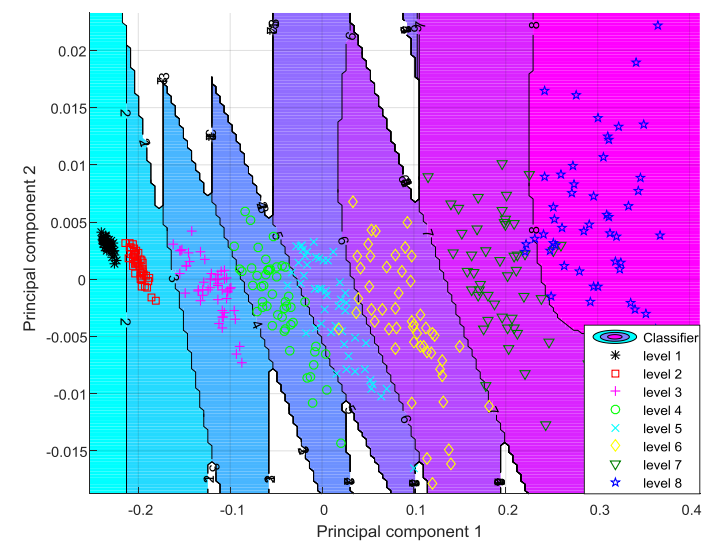

(a) Training features with linear kernel PCA

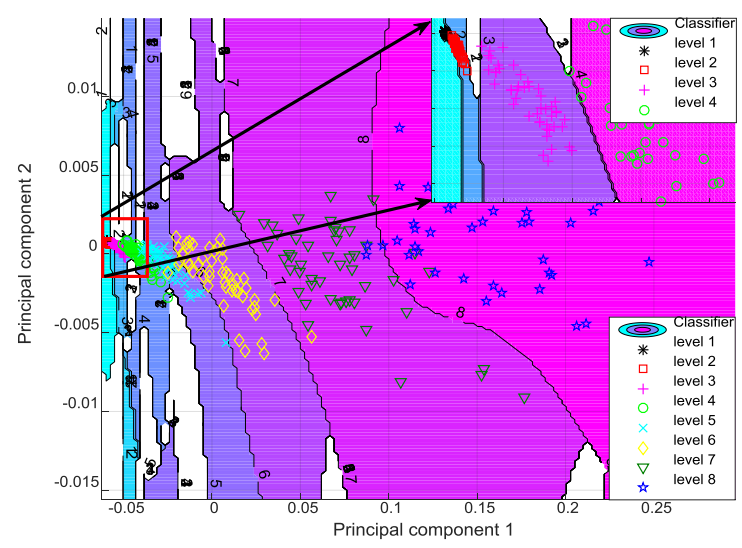

(b) Training features with polynomial kernel PCA 


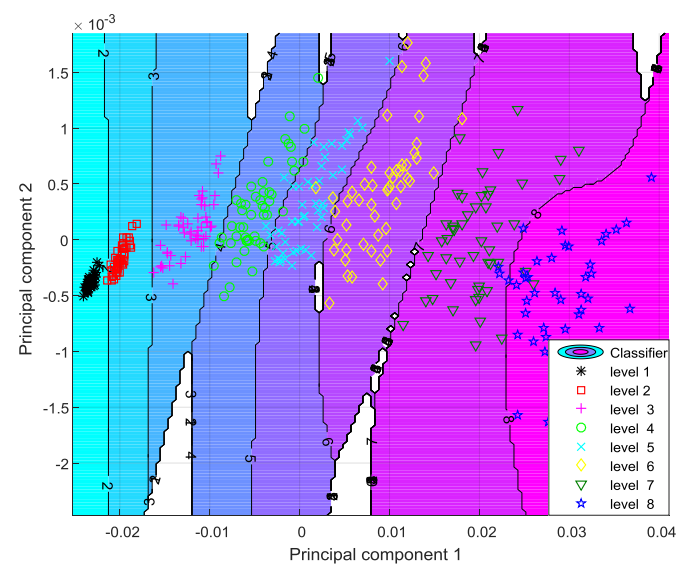

(c) Training features with RBF kernel PCA

Fig.6 Class separation lines of training features used SVM classifier : (a) training features with linear kernel PCA, (b) training features with polynomial kernel PCA, (c) training features with RBF kernel PCA

To conduct a comprehensive investigation of the SVM classifier, results using different classifiers, including the $k$-nearest neighbor classifier $(k-\mathrm{NN})$, neural network classifier $(\mathrm{NN})$, naive Bayes classifier $(\mathrm{NB})$ and decision tree classifier (DT), are shown in Tables4 6.

Table 4 Leakage level recognition using kernel PCA with linear kernel function

\begin{tabular}{|c|c|c|c|c|c|}
\hline \multirow[t]{2}{*}{ Classifier } & \multicolumn{3}{|c|}{ Training } & \multicolumn{2}{|c|}{ Testing } \\
\hline & $\begin{array}{l}\text { Accuracy } \\
(\%)\end{array}$ & $\begin{array}{l}\text { Cohen's } \\
\text { kappa }\end{array}$ & $\begin{array}{l}\text { Training time } \\
\text { (s) }\end{array}$ & $\begin{array}{l}\text { Accuracy } \\
(\%)\end{array}$ & $\begin{array}{l}\text { Cohen's } \\
\text { kappa }\end{array}$ \\
\hline SVM & 96.75 & 0.96 & 58.00 & 95.00 & 0.94 \\
\hline$k-N N(k=10)$ & 88.00 & 0.86 & 0.22 & 88.25 & 0.87 \\
\hline NN & 65.25 & 0.60 & 12.97 & 72.50 & 0.69 \\
\hline NB & 80.00 & 0.77 & 0.39 & 84.25 & 0.82 \\
\hline DT & 91.25 & 0.90 & 0.08 & 85.50 & 0.83 \\
\hline
\end{tabular}

Table 5 Leakage level recognition using kernel PCA with polynomial kernel function

\begin{tabular}{llllll}
\hline Classifier & \multicolumn{3}{c}{ Training } & Testing \\
\hline & Accuracy & Cohen's & Training & Accuracy & Cohen's \\
& $(\%)$ & kappa & time $(\mathrm{s})$ & $(\%)$ & kappa \\
SVM & 96.75 & 0.96 & 58.92 & 94.25 & 0.93 \\
k-NN $(\mathbf{k = 1 0})$ & 86.25 & 0.84 & 0.03 & 88.00 & 0.86 \\
NN & 42.00 & 0.34 & 13.28 & 43.00 & 0.35 \\
NB & 81.00 & 0.78 & 0.25 & 82.00 & 0.80 \\
DT & 74.75 & 0.71 & 0.05 & 77.25 & 0.74 \\
\hline
\end{tabular}

Table 6 Leakage level recognition using kernel PCA with RBF kernel function

\begin{tabular}{llllll}
\hline Classifier & \multicolumn{3}{c}{ Training } & \multicolumn{2}{c}{ Testing } \\
\hline & Accuracy & Cohen's & Training & Accuracy & Cohen's \\
& $(\%)$ & kappa & time (s) & $(\%)$ & kappa \\
\hline
\end{tabular}




\begin{tabular}{llllll}
\hline SVM & 96.50 & 0.96 & 61.23 & 95.25 & 0.95 \\
k-NN (k=10) & 87.75 & 0.86 & 0.03 & 89.25 & 0.88 \\
NN & 45.75 & 0.39 & 12.59 & 47.75 & 0.41 \\
NB & 80.25 & 0.77 & 0.25 & 82.75 & 0.80 \\
DT & 90.75 & 0.89 & 0.06 & 90.75 & 0.89 \\
\hline
\end{tabular}

The experimental results of the SVM classification using kernel PCA feature dimensionality reduction are shown in Tables 4 6, it can also be seen that the SVM is the optimal classifier to recognize the levels of the pipeline valve leakage with a higher accuracy and a better agreement in the Cohen's kappa between the data of the training results and the corresponding testing process. The accuracy and Cohen's kappa of the training process reach up to $96.75 \%$ and 0.96 , while the two parameters are $95.25 \%$ and 0.95 in the testing process, respectively. However, the training time for the classification process using the SVM takes longer than for the other classifiers. This is because the cross-validation method is adopted to determine the optimal penalty factor $C$ and kernel parameter $\gamma$. Consequently, the structure of kernel PCA and SVM leads to the highest efficiency, accuracy, and Cohen's kappa to recognize the different leakage levels of the valve in the gas pipeline.

\section{Conclusions}

In this study, acoustic emission technology was used to obtain the acoustical signal of the gas pipeline valve leakage, and the measured $\mathrm{AE}$ parameters in the time and frequency domains were used as leakage features. The application of nonlinear feature dimensionality reduction and SVM for recognizing the leakage levels was proposed. It was demonstrated by the experiment that the kernel PCA could be effectively used for the reduction of the feature dimensionality based on the mapping of a set of 8-D feature vectors to a new 2-D space, and the recognition performance obtained by SVM was higher than those by the other classifiers. The optimal values of the penalty factor $C$ and kernel parameter $\gamma$, which were selected by cross-validation, were critical to improve the performance and accuracy of the SVM model. The experimental results indicated that the optimum algorithm, which is composed of the kernel PCA and SVM, led to the highest recognition performance of the leakage levels, with an accuracy of $96.75 \%$ and a Cohen's kappa of 0.96 for the training process. This is very close to the corresponding values of $95.25 \%$ and 0.95 in the testing process. Consequently, the intelligent recognition of acoustic emission using the kernel PCA and SVM model was proven to be an effective tool to recognize the leakage levels of gas pipeline valves.

\section{Acknowledgements}


The work was supported by the National Science Foundation of China (Grant No. 51106180) and the Scientific Research Project Foundation of Petro-China (Grant No. 2012E-2802).

\section{References}

Burges C J C. A tutorial on support vector machines for pattern recognition [J]. Data mining and knowledge discovery, 1998, 2 (2): 121-167.

Cho J H, Lee J M, Choi S W, et al. Fault identification for process monitoring using kernel principal component analysis [J]. Chemical engineering science, 2005, 60 (1): 279-288.

Choi S. Detection of valvular heart disorders using wavelet packet decomposition and support vector machine [J]. Expert Systems with Applications, 2008, 35 (4): 1679-1687.

Christian G, Masayasu O. Acoustic emission testing [M]. Springer Science \& Business Media, 2008..

Cohen J. A coefficient of agreement for nominal scales [J]. Educational and Psychosocial Measurement 1960, 20, $37-46$.

Cortes C, Vapnik V. Support-vector networks [J]. Machine learning, 1995, 20 (3): 273-297.

Ding W, Tong Y, Zhang Q, et al. Image and video quality assessment using neural network and svm [J]. Tsinghua Science \& Technology, 2008, 13(1): 112-116.

Drouillard T F. A history of acoustic emission [J]. Journal of acoustic emission, 1996, 14 (1): 1-34.

Gil D, Johnsson M. Using support vector machines in diagnoses of urological dysfunctions [J]. Expert Systems with Applications, 2010, 37 (6): 4713-4718.

Hsu C W, Lin C J. A comparison of methods for multiclass support vector machines [J]. Neural Networks, IEEE Transactions on, 2002, 13 (2): 415-425.

Jiang L, Cai Z, Wang D, et al. Improving tree augmented naive bayes for class probability estimation [J]. Knowledge-Based Systems, 2012, 26: 239-245.

Kaewwaewnoi W, Prateepasen A, Kaewtrakulpong P. Investigation of the relationship between internal fluid leakage through a valve and the acoustic emission generated from the leakage [J]. Measurement, 2010, 43 (2): 274-282.

Kulkarni A, Jayaraman V K, Kulkarni B D. Knowledge incorporated support vector machines to detect faults in Tennessee Eastman Process[J]. Computers \& chemical engineering, 2005, 29 (10): 2128-2133.

Lee J H, Lee M R, Kim J T, et al. A study of the characteristics of the acoustic emission signals for condition monitoring of check valves in nuclear power plants [J]. Nuclear engineering and design, 2006, 236 (13): 
1411-1421.

Liao Y, Vemuri V R. Use of k-nearest neighbor classifier for intrusion detection [J]. Computers \& Security, 2002, 21 (5): 439-448.

Malhotra V M, Carino N J. Handbook on Nondestructive Testing of Concrete Second Edition [M]. CRC press, 2003.

Meland E, Henriksen V, Hennie E, et al. Spectral analysis of internally leaking shut-down valves [J]. Measurement, 2011, 44 (6): 1059-1072.

Noipitak M, Prateepasen A, Kaewwaewnoi W. A relative calibration method for a valve leakage rate measurement system [J]. Measurement, 2011, 44 (1): 211-218.

Oh C K, Sohn H. Damage diagnosis under environmental and operational variations using unsupervised support vector machine [J]. Journal of Sound and Vibration, 2009, 325 (1): 224-239.

Prateepasen A, Kaewwaewnoi W, Kaewtrakulpong P. Smart portable noninvasive instrument for detection of internal air leakage of a valve using acoustic emission signals [J]. Measurement, 2011, 44 (2): 378-384.

Schölkopf B, Smola A, Müller K R. Nonlinear component analysis as a kernel eigenvalue problem [J]. Neural computation, 1998, 10 (5): 1299-1319.

Sethi I K. Structure-driven induction of decision tree classifiers through neural learning [J]. Pattern Recognition, 1997, 30 (11): 1893-1904.

Sharif M A, Grosvenor R I. Internal valve leakage detection using an acoustic emission measurement system [J]. Transactions of the Institute of Measurement and Control, 1998, 20(5): 233-242.

Tohmé M, Lengellé R. Maximum margin one class support vector machines for multiclass problems [J]. Pattern recognition letters, 2011, 32 (13): 1652-1658.

Tsujinishi D, Abe S. Fuzzy least squares support vector machines for multiclass problems [J]. Neural Networks, 2003, 16 (5): 785-792.

Yang B S, Hwang W W, Ko M H, et al. Cavitation detection of butterfly valve using support vector machines [J]. Journal of sound and vibration, 2005, 287 (1): 25-43.

Yu Y, Junsheng C. A roller bearing fault diagnosis method based on EMD energy entropy and ANN [J]. Journal of sound and vibration, 2006, 294 (1): 269-277. 
Tel: +86-18810114818; fax: +86-10-89733203.

E-mail address: haifengzhang1986@163.com.

Address: PetroChina Pipeline R\&D Center, No.51, Jin-guang Road, Langfang, Hebei 065000, China. 